\title{
Development of a Troponin-Specific Probe for Early Detection of Cardiac Injuries
}

\author{
Lara Pes ${ }^{1, \S}$, Young Kim ${ }^{1,2, \S}$, and Ching-Hsuan Tung, ${ }^{1, *}$ \\ ${ }^{I}$ Molecular Imaging Innovations Institute, Department of Radiology, Weill Cornell Medical College, \\ New York, NY, 10021, USA; ${ }^{2}$ Department of Pathology, Chonnam National University \\ Medical School, 671, Jebongno, Dong-Gu, Gwangju, 501-757, Korea \\ ${ }^{\S}$ These authors contributed equally to the development of the project.
}

\section{Introduction}

Cardiac troponin I (cTnI) is an important cardiomyocyte-specific protein responsible for heart muscle contraction. It is released into the bloodstream subsequently to heart damage; for this reason, it is widely used as a biomarker in the early detection of myocardial injuries [1,2]. Recently a novel peptide termed myocyte targeting peptide (MTP, Figure 1), which has unusual affinity to cardiomyocytes was identified by us $[3,4]$. This prototype MTP contains three residues of the non-natural amino acid biphenylalanine (Bip) and its selectivity towards cardiac tissues is thought to be due to the ability of Bip to bind cTnI. With the aim of early heart injury diagnosis, a library of MTP derivatives was designed in order to optimize the affinity and specificity of the troponin binding. Assisted by a fluorescein (FITC) label, the specific selectivity of the MTP analogs to myocardium over skeletal muscle tissues was assayed by fluorescence imaging. The improved MTP could potentially be used to detect circulating cTnI after cardiac injury.

\section{Results and Discussion}

Despite the good results previously obtained with MTP in selectively binding cTnI in cardiac muscle tissues, a new library of derivatives was developed (Figure 2) in order to investigate the role of the unusual Bip amino acid and the number of phenyl groups in the peptide structure and better understand structure-activity relationship. MTP peptides were prepared on Rink Amide MBHA resin using Fmoc chemistry, HBTU and piperidine were employed as coupling reagent and deprotecting agent respectively. All the compounds were labeled with a FITC on their termini. Troponin specific binding was performed on sliced frozen sections of cardiac and skeletal muscle tissue (the latter used as a control) for $1 \mathrm{~h}$ with the MTP derivatives in PBS, at concentrations from 2 to $10 \mu \mathrm{M}$. Fluorescence images were taken and the fluorescence intensity was calculated using the software Image J.

To evaluate the ability of the Bip amino acid alone to interact with cTnI, the fluorescent tag was grafted directly to its $N$ terminus, with and without a spacer (BipHA and BipAA respectively), but in tissue study no fluorescence was registered, indicating that Bip alone does not provide a strong enough

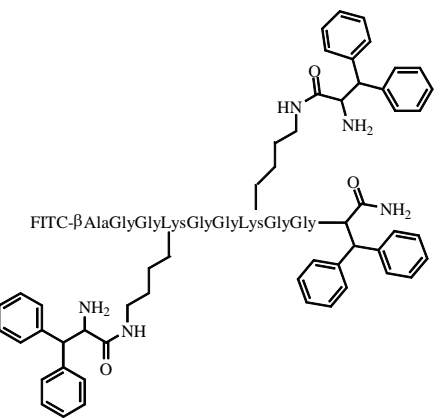

Fig. 1. Structure of MTP. binding. A Bip dimer was then prepared and a short peptide chain was added (sBip2) to improve solubility and mimic the original MTP, in which the Bip are part of a peptide structure. The newly prepared sBip2 was able to preferentially stain cardiac muscles over skeletal muscle tissues. At the same time, other analogs of sBip2 were synthesized aiming to understand the importance of the number of phenyl groups; in sPheBip and sGlyBip, one Bip was replaced with a phenylalanine or glycine, bringing the number of aryls from four to three and two respectively and in sPhePhe, both Bip residues were substituted with phenylalanine. Consistent with previous findings, a decrease in the ability of staining cardiac muscle with a decrease in the number of phenyl groups was observed. In the case of sGlyTrip, where a triphenyl moiety was used, the selectivity towards cardiac muscles was lost. Finally the PEG5Bip2 analog, synthesized in solution over 5 steps, substituting the Bip amino acid with Bip amine and the peptide chain with an equivalent length PEG, showed binding, but no selectivity. 
Among the first series of compounds, the best results in fluorescence intensity and selectivity were obtained with sBip2, but still the solubility was not as hoped. A positively charged poly-arginine chain was thus introduced to improve solubility and, as expected, both Bip1 and Bip2 were fully soluble. In addition, Bip1 and Bip2, which have one and two Bip amino acid residues respectively, showed significantly improved binding to cardiac muscle tissues and selectivity over skeletal muscles. Bip2 was found to be the best derivative from this set of the library (Figure 3).
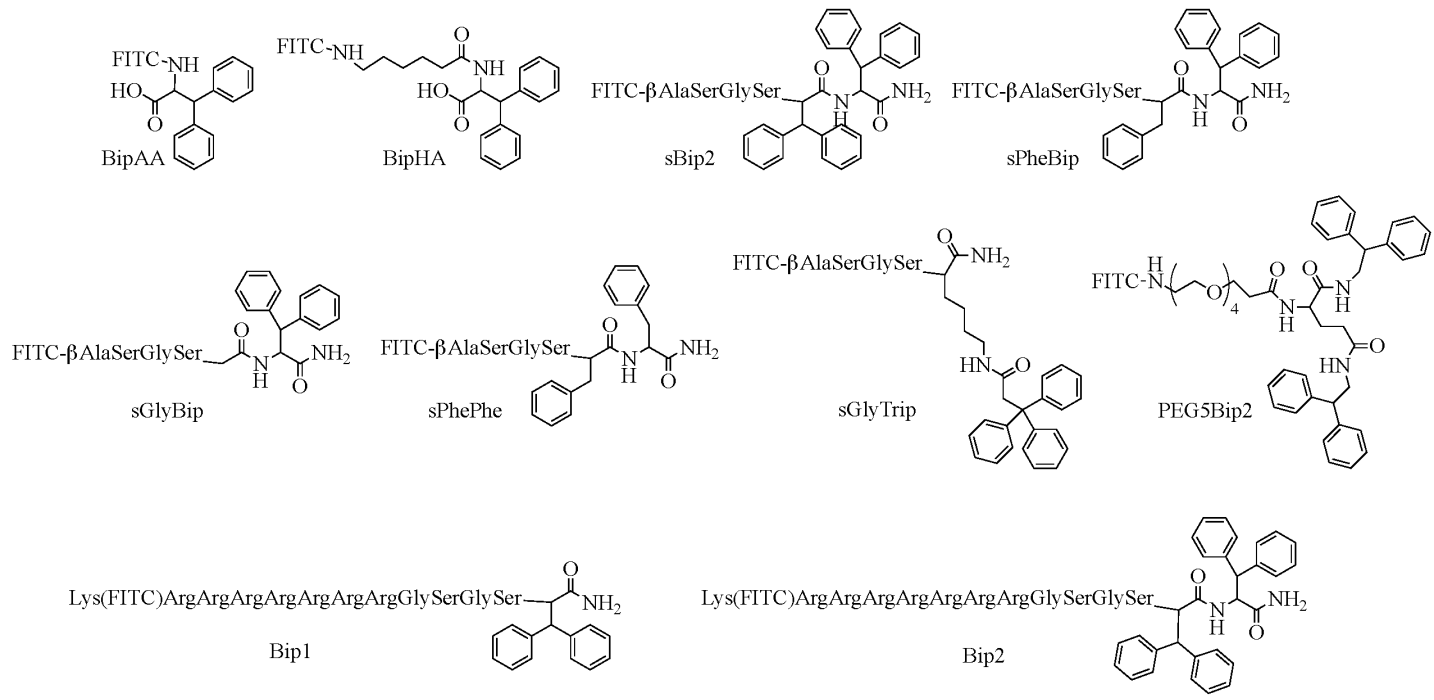

Fig. 2. Structures of the MTP derivatives.

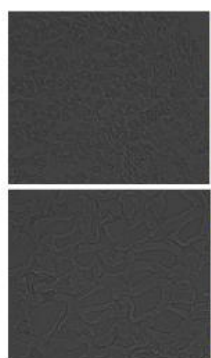

TRANS
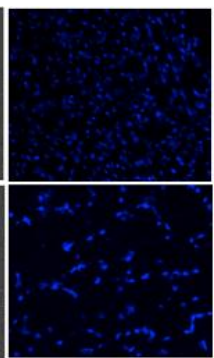

DAPI

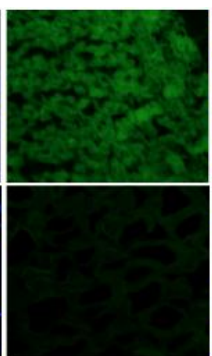

FITC

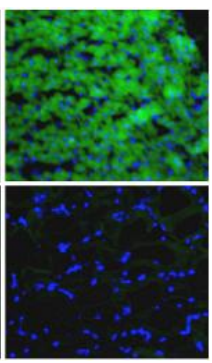

MERGE

Fig. 3. Fluorescence microscope images, $20 x$ at $30 \mathrm{~ms}$ exposure time, of frozen sections of cardiac muscles (top) and skeletal muscles (bottom) after 1 h staining with $2 \mu M$ solutions in PBS of the most active MTP derivative Bip2.

The presence of the phenyl groups proved to be important in cTnI binding, but the peptide chain also played a critical role. The biphenylalanine alone shows no activity, yet the addition of the polyarginine sequence leads to improvements in its binding and selectivity. Currently new derivatives are being developed to investigate the effect of the polyarginine sequence and length of the peptide.

\section{References}

1. De Lemos, J.A. JAMA 309, 2262-2269 (2013), http://dx.doi.org/ 10.1001/jama.2013.5809

2. Mahajan, V.S., Jarolim, P. Circulation 124, 2350-2354 (2011),

http://dx.doi.org/10.1161/CIRCULATIONAHA.111.023697

3. Chen, J., et al. PLoS ONE 6, e19097 (2011), http://dx.doi.org/10.1371/journal.pone.0019097

4. Abd-Elgaliel, W.R., Tung, C.H. Mol. Biosyst. 8, 2629-2632 (2012), http://dx.doi.org/10.1039/c2mb25178g 